\title{
Eficiencia en la producción porcina en países de Europa. Casos de Dinamarca y Polonia
}

\author{
Efficiency in swine production in European countries. Cases of Denmark and Poland
}

\author{
Juan Cabas-Monje ${ }^{1 *}$, Luis Améstica-Rivas², Jonathan Labra-Hernández², Bouali Guesmi ${ }^{3}$ y José María Gil ${ }^{3}$ \\ ${ }^{1}$ Facultad de Ciencias Empresariales, Departamento de Gestión Empresarial, Grupo de Investigación en Agronegocios, Universidad \\ del Bío-Bío. Región de Nuble, Chile. ${ }^{2}$ Facultad de Ciencias Empresariales, Departamento de Gestión Empresarial, Universidad del \\ Bío-Bío. Región de Ñuble, Chile. ${ }^{3}$ Center for Agro-Food Economics and Development (CREDA-UPC-IRTA). Castelldefels, España. \\ *Correo electrónico: jcabas@ubiobio.cl
}

\begin{abstract}
RESUMEN
La carne de cerdo $(\mathrm{CdC})$ es el segundo tipo de carne producido en el mundo, siendo Europa la segunda región en importancia productiva después de Asia. Dentro del mercado europeo de CdC, Dinamarca y Polonia son dos actores relevantes que presentan algunas diferencias en sus sistemas productivos (SP). El objetivo fue estudiar las variables que explican el comportamiento de la producción de carne porcina y la eficiencia técnica relativa de los SP tomando en cuenta las diferencias en los sistemas. Se utilizó modelos de frontera estocástica de producción del tipo Translog, para muestras de productores porcinos en el periodo 2010-2015. Los resultados indican que las muestras de productores de ambos países presentan niveles de eficiencia altos, con valores de 0,899 y 0,859 para Dinamarca y Polonia, respectivamente. Los factores más importantes que explican la producción de cerdos son los alimentos y el capital. Además, Polonia presenta crecimiento tecnológico significativo en el periodo estudiado sin mostrar cambios en la eficiencia, mientras que, Dinamarca no presenta progreso tecnológico y adicionalmente muestra una baja en la eficiencia técnica relativa en el periodo.
\end{abstract}

Palabras clave: Eficiencia técnica; frontera estocástica; producción porcina

\begin{abstract}
Pork is the second type of meat produced in the world, with Europe being the second most important Region after Asia. Within the European pork meat market, Denmark and Poland are two relevant players with some differences in their production systems (PS). The objective of this research was to study the variables that explain the behavior of pig meat production and the relative technical efficiency of the PS, taking into account the differences in the systems. Stochastic production frontier models of the Translog type were used for samples of swine producers in the period 20102015. The results indicate that the samples of producers from both Countries present high levels of efficiency, with values of 0.899 and 0.859 for Denmark and Poland, respectively. The most important factors that explain pig production are food and capital. In addition, Poland presents significant technological growth in the period without changes in efficiency, while Denmark does not present significant technological progress and a decrease in relative technical efficiency in the period.
\end{abstract}

Key words: Technical efficiency; stochastic frontier; pig production 


\section{INTRODUCCIÓN}

La CdC (Sus scrofa domestica) es el segundo tipo de carne más producida en el mundo tras la carne de ave, con un pronóstico de una producción de 101 millones de toneladas (Mill Ton) para el año 2020, abarcando con ello aproximadamente el $30 \%$ de la producción mundial de carne. Europa es la segunda región de mayor producción de carne porcina a nivel mundial, después de Asia, concentrando el 27,1\% del mercado global [15]. La producción en Europa es liderada por Alemania con un total de $21,9 \%$ de la producción de la Comunidad Europea (CE), seguida por España con el 19,5 \% y Francia con el 9,2 \%. Polonia es el cuarto mayor productor en la región con un 8,3 \% de la producción del año 2019, mientras que, Dinamarca está en el sexto lugar con un $6,3 \%$ [25].

Dinamarca y Polonia, además de ser importantes en el mercado de carne porcina presentan ciertas características particulares en su sistema productivo (SP) y comercializador, que resulta de interés estudiarlo. En Dinamarca, la industria porcina desempeña un papel relevante en la economía local, donde más del $90 \%$ de su producción se exporta a 120 mercados de todo el mundo, esto representa más del $19 \%$ del total de productos alimentarios exportados [11]. Si se consideran los valores productivos en términos per-cápita, Dinamarca es la mayor potencia en el mundo. Lidera en rendimiento por lechones destetados por cada cerda en reproducción al año(a) y tiene un SP con el más avanzado desarrollo tecnológico, bajo estricta legislación ambiental y sanitaria, y concentrado principalmente en grandes explotaciones. Tiene una estructura cooperativa, en la que los criadores son propietarios de la producción, los mataderos y las empresas de elaboración de alimentos. Es decir, presentan una estructura totalmente integrada [14].

Polonia ha experimentado cambios estructurales importantes en las últimas dos décadas, aumentando la concentración de la producción en granjas de mayor tamaño. Pero, en comparación con otros países de la Unión Europea, la producción sigue presentando una estructura muy fragmentada, con escalas de producción muy bajas, lo que lleva a importar grandes cantidades de lechones para la cría y carne principalmente desde Dinamarca. A lo anterior, se suma el hecho de una integración vertical y horizontal poco desarrollada, con escasa colaboración entre productores de cerdos y procesadores de carne [19, 26].

Aún cuando Polonia incrementó su participación en el mercado europeo, pasando del 7,8 \% en 2010 a 8,3 \% en 2019, Dinamarca la disminuyó de un $7,5 \%$ a un $6,3 \%$ en 2019 . Se considera a Dinamarca como un referente mundial en términos de su SP y en su estructura cooperativa, y a Polonia como un país que debe mejorar para alcanzar niveles de desarrollo superiores [25].

Como una forma de profundizar en el conocimiento de los SP de cerdos de ambos países, esta investigación tuvo como propósito estudiar las variables que explican el comportamiento de la producción de carne porcina y la eficiencia técnica relativa de los SP, de tal manera de contribuir a la discusión sobre la eficiencia técnica en la producción porcina, considerando que la literatura en este tema es escasa y donde en las estimaciones se han utilizado, tanto las aproximaciones paramétricas de frontera estocástica [17, 18, 30, 31], como no paramétricas (Análisis Envolvente de Datos, DEA) [7] y Análisis de Eficiencia Multidireccional (MEA) [23]. Es decir, la eficiencia técnica se debe entender como la capacidad de la empresa para utilizar de mejor forma posible los recursos disponibles para alcanzar un determinado nivel de producción [13]. En particular, se quiere determinar la relevancia de los factores productivos como trabajo, capital, alimento, costos de insumos y progreso tecnológico en la producción porcina en ambos países, así como el nivel de eficiencia de los productores y su evolución en el tiempo.

\section{MATERIALES Y MÉTODOS}

\section{Producción de carne de cerdo}

La producción de CdC es la segunda a nivel mundial después de la producción de carne de ave. En el año 2018, la producción total de carne alcanzó los 342,2 Mill Ton, de las cuales, 127,3 Mill Ton correspondió a carne de ave y 120,9 Mill Ton fueron de CdC [15]. En tercer lugar, está la producción de carne bovina (Bos taurus) y en cuarto lugar la producción de ovino (Ovis aries). Es importante mencionar que, las estimaciones realizadas por la Organización de las Naciones Unidas para la Alimentación y la Agricultura (FAO) señalan que por segundo año consecutivo, la producción mundial de carne caerá, alcanzando 333 Mill Ton en el año 2020, lo que corresponde a un 1,7 \% menos que la estimación del año 2019 [15]. Una explicación de esto sería la fuerte caída en la producción de carne porcina, concentrada principalmente en los países asiáticos afectados por la peste porcina africana [15].

Por otra parte, la expansión de la producción de carne ha sido afectada negativamente por la pandemia del COVID-19, que ha impactado a la sociedad mundial. Los efectos de la crisis sanitaria y económica han repercutido, tanto en la oferta como en la demanda de productos cárnicos. Los consumidores están evaluando sus prácticas de compra y consumo de carne poniendo cada vez más atención a los temas de salud, seguridad alimentaria, sustentabilidad, confianza y valor. El desempleo, la recesión económica y la disminución de la actividad de hotelería y restauración ha afectado directamente la compra de carne. A lo anterior se suma el distanciamiento social, el cierre de los mercados locales y la escasez de trabajadores en los mataderos y las plantas procesadores ha influido negativamente en la cadena de elaboración y en los niveles de producción [15].

Asia fue la región con mayor producción de CdC, abarcando el $49,6 \%$ de la producción mundial según una estimación de FAO [15]. China es el principal país productor con un total de 43.447 Mill Ton para el año 2019. Europa es la segunda región de mayor producción de carne porcina a nivel mundial, concentrando el $27,1 \%$ del mercado global. Esta producción es liderada por los países de la Unión Europea junto al Reino Unido. No obstante, dado los cambios experimentados en el mercado de la carne derivados del COVID-19, se proyecta para el año 2020 que la producción asiática disminuirá en cerca de 9.000 Mill Ton [15].

Respecto a producción de CdC para los países de la Unión Europea (incluido Reino Unido), Alemania corresponde al principal país productor de CdC en el año 2019 con un total de 21,9\% de la producción de la Comunidad Europea (CE), seguida por España con el $19,5 \%$ y Francia con el 9,2 \%. Polonia es el cuarto mayor productor en la región con un 8,3 \%, mientras que, Dinamarca está en el sexto lugar con un 6,3\%. Polonia ha incrementado su participación en el mercado regional pasando del 7,8 \% en 2010 al 8,3 \% en 2019. Por el contrario, Dinamarca ha disminuido 
su participación en la producción de CdC en la Unión Europea pasando de un 7,5 a un $6,3 \%$ en 2019 [25].

\section{Eficiencia en la producción}

En el estudio de eficiencia existen diversas metodologías que permiten analizar el SP. Farrell [13] fue un pionero en este campo y a partir de su trabajo se han diseñado nuevas herramientas de análisis, entre ellas destacan el Análisis Envolvente de Datos (Data Envelopment Analysis, DEA por su sigla en inglés) como una aproximación no paramétrica [8], su modificación denominada Análisis de Eficiencia Multidireccional (Multidirectional efficiency analysis, MEA por su sigla en inglés) [2] o el Análisis de Fronteras Estocásticas (Stochastic Frontier Analysis, SFA por su sigla en inglés), en el cual se suele utilizar una forma funcional del tipo CobbDouglas y una metodología de estimación de Máxima Verosimilitud (MV) para medir la eficiencia [20, 27] o una especificación del tipo Translog con el método de MV [6], presentando ambos métodos (DEA y SFA) fortalezas y debilidades [5, 32].

En el ámbito de la investigación en producción de carne, por medio de una aproximación no paramétrica (DEA) se mide la eficiencia en la producción de porcinos y aves de engorde en el sector ganadero de Valencia, España [7]. El estudio concluye que las granjas de la comunidad Valenciana tienen un potencial significativo de mejora en ambos rubros, constatando que solo un $5,1 \%$ de las granjas de producción de cerdo son completamente eficientes.

En tanto, en una investigación para estudiar la eficiencia técnica en la producción de cerdo en Suecia se utilizó el análisis multidireccional [23]. Este estudio se centró en el proceso de producción dividido en las fases de: i) lechón, ii) crecimientofinalización y iii) finalización-parto, enfocándose en la eficiencia técnica de cada una de las entradas y salidas identificadas. Los resultados indican que las variables de instrucciones escritas para alimentación en la etapa de crecimiento-finalización y finalización-parto, así como las instrucciones escritas para prevenir enfermedades infecciosas en lechones, constituyen las variables con mayor contribución a la eficiencia técnica en producción de cerdos.

Por otra parte, en Nigeria utilizan una función de producción de frontera estocástica para estudiar la producción de cerdo [31]. En esta investigación se emplean variables de producción y características de los productores para estimar sus efectos en el SP. Los resultados muestran que la educación del productor contribuye positivamente a la eficiencia. Así también variables como insumos, mano de obra, medicamentos y vacunas tienen efectos positivos significativos en la producción, alcanzando una eficiencia técnica media de 0,97.

\section{Frontera estocástica de producción}

A fin de capturar los shocks exógenos que afectan a una empresa e identificar los errores de medición que pueden producirse, surge el modelo de frontera estocástica (MFE) de producción o modelo de error compuesto, el cual es una técnica estadística para estimar parámetros [1,24], en los que el error está compuesto de dos partes: i) un componente simétrico que captura los errores de medición además de los shocks externos, permitiendo una variación estocásticas, y ii) un componente unilateral que captura la ineficiencia [16].
La descripción del MFE junto con ejemplos aplicados en agricultura pueden ser encontrados en varios trabajos [10, 21, 22]. Un número considerable de estos estudios empíricos en agricultura emplean un SFA con forma funcional Cobb-Douglas, no obstante, también se puede suponer otra forma funcional como CES o elasticidad de sustitución, translogarítmica, entre otras.

La frontera estocástica de producción (FEP) está definida en la ecuación (1):

$$
\begin{aligned}
& Y_{i}=f\left(X_{i} ; \beta\right) e^{v-u} \\
& \text { Donde } v \sim N\left(0, \sigma_{v}^{2}\right), u \sim N^{+}\left(0, \sigma_{u}^{2}\right), i=1,2, \ldots, n .
\end{aligned}
$$

En la ecuación (1) $Y_{i}$ es la producción de CdC de cada individuo $i, X_{i}$ es el vector de insumos del productor $i, \beta$ son los parámetros de factores estimados. La función de producción es creciente y obligatoriamente cóncava, y si la producción real se desvía de la teóricamente posible, $-u \leq 0$ explica la ineficiencia técnica y $v$ explica el ruido estadístico, ambos independientes entre sí. Sí $u=0$ la unidad de estudio es eficiente, mientras que si $-u<0$ entonces existe ineficiencia y su distribución se asume semi-normal, truncada en 0 con una distribución concentrada en el semi-intervalo [0,infinito] [1, 5, 10, 24].

El método de máxima verosimilitud se propone para la estimación simultánea de los parámetros de la frontera estocástica y el modelo de ineficiencia. Los parámetros de varianza de la función de máxima verosimilitud son estimados a partir del modelo de varianza definido como $\sigma_{s}^{2}=\sigma_{v}^{2}+\sigma_{u}^{2}$, insumo para determinar el parámetro $\gamma=\frac{\sigma_{u}^{2}}{\sigma_{v}^{2}}$,el cual considera la proporción de la varianza total que es explicado por la varianza de las ineficiencias y considera valores entre 0 y 1 , en su descripción además se considera el parámetro $\lambda=\sqrt{\frac{\sigma_{u}^{2}}{\sigma_{v}^{2}}}$ que da una relación entre las varianzas componentes del error .

La eficiencia técnica (ET) puede ser medida a través de cociente entre la producción observada versus la FEP, como muestra la ecuación (2):

$$
E T=\frac{y}{f(x) e^{v}}=\frac{f(x) e^{-u} e^{v}}{f(x) e^{v}}=e^{-u}
$$

\section{Eficiencia con datos de panel y cambios en el tiempo}

Henningsen [17], siguendo el modelo desarrollado por Battese y Coelli [3], plantea que la función de frontera de producción con cambio tecnológico se puede estimar de diferentes maneras, sin embargo, todas derivan de la ecuación (3) que corresponde a su fórmula general

$$
\ln y_{k t}=\ln f\left(x_{k t}, t\right)-u_{k t}+v_{k t}
$$

donde el subíndice $k=1, \ldots, K$ indica la empresa, $t=1, \ldots, T$ indica el periodo de tiempo, y las otras variables son definidas como se mencionaron previamente. De esto se pueden obtener tres variantes que se especifican a continuación:

1.- Eficiencias individuales invariantes en el tiempo (timeinvariant), es decir, $u_{k t}=u_{k}$, lo que significa que cada empresa tiene una eficiencia fija individual que no varía en el tiempo;

2.- Eficiencias individuales variables en el tiempo (time-variant), es decir, $u_{k t}=u_{k} \exp (-\eta(t-T))$, lo que significa que cada empresa tiene una eficiencia individual y los términos de eficiencia 
de todas las empresas puede variar con el tiempo con la misma tasa (y en la misma dirección); y

3.- Eficiencias específicas de la observación, es decir, sin restricciones sobre $u_{k t}$, lo que significa que el término de eficiencia de cada observación se estima independientemente de las otras eficiencias de la empresa, de modo que básicamente se ignora la estructura de panel de los datos.

De tal forma, en esta investigación se utilizó una aproximación paramétrica de FEP para datos de panel de segunda generación, desarrollados por Battese y Coelli $[3,4]$ que se muestra en la ecuación (4) para estudiar la eficiencia en la producción de CdC en Polonia y Dinamarca. La estimación de los parámetros se realiza utilizando "Frontier Package 1.1-8" en R versión 3.6.3 [9, 28].

$$
\begin{aligned}
& \ln y_{k t}=\beta_{0}+\beta_{1} \ln \left(\text { feed }_{k t}\right)+\beta_{2} \ln \left(o i_{k t}\right)+\beta_{3} \ln \left(\operatorname{labor}_{k t}\right)+\beta_{4} \ln \left(\operatorname{cap1}_{k t}\right) \\
& +\beta_{5} \ln \left(\operatorname{cap} 2_{k t}\right)+\beta_{6} m \text { Year }+\beta_{11}\left(\ln \left(\text { feed }_{k t}\right)\right)^{2} / 2 \\
& +\beta_{22}\left(\ln \left(\text { oi }_{k t}\right)\right)^{2} / 2+\beta_{33}\left(\ln \left(\text { labor }_{k t}\right)\right)^{2} / 2 \\
& +\beta_{44}\left(\ln \left(\operatorname{cap} 1_{k t}\right)\right)^{2} / 2+\beta_{55}\left(\ln \left(\operatorname{cap} 2_{k t}\right)\right)^{2} / 2 \\
& +\beta_{12} \ln \left(\text { feed }_{k t}\right) \ln \left(\text { oi }_{k t}\right)+\beta_{13} \ln \left(\text { feed }_{k t}\right) \ln \left(\text { labor }_{k t}\right) \\
& +\beta_{14} \ln \left(\text { feed }_{k t}\right) \ln \left(\operatorname{cap}_{k t}\right)+\beta_{15} \ln \left(\text { feed }_{k t}\right) \ln \left(\operatorname{cap} 2_{k t}\right) \\
& +\beta_{23} \ln \left(o i_{k t}\right) \ln \left(\text { labork }_{k t}\right)+\beta_{24} \ln \left(o i_{k t}\right) \ln \left(\operatorname{capl}_{k t}\right) \\
& +\beta_{25} \ln \left(\text { oi }_{k t}\right) \ln \left(\operatorname{cap} 2_{k t}\right)+\beta_{34} \ln \left(\operatorname{labor}_{k t}\right) \ln \left(\operatorname{cap} 1_{k t}\right) \\
& +\beta_{35} \ln \left(\text { labor }_{k t}\right) \ln \left(\operatorname{cap}_{k t}\right)+\beta_{45} \ln \left(\operatorname{cap} 1_{k t}\right) \ln \left(\operatorname{cap} 2_{k t}\right) \\
& +\theta t+v_{k t}-u_{k t}
\end{aligned}
$$

\section{Descripción de variables utilizadas}

Los datos de producción porcina son obtenidos desde la Farm Accountancy Data Network (FADN) para el periodo 2010 al 2015, constituyendo un panel no balanceado con 91 empresas de Polonia y 92 empresas de Dinamarca, con un total de 396 y 358 observaciones, respectivamente. La TABLA I muestra el resumen de las variables utilizadas por año para Polonia y Dinamarca. La variable dependiente o explicada es la producción de cerdos medida como el ingreso total en euros. Se definen cinco factores productivos o insumos, el capital1, el capital2, el trabajo, alimento $\mathrm{y}$ otros insumos.

El capital1 comprende edificios, equipamiento, máquinas, tractores, etc. El capital2 incluye el número de equinos (Equus caballus), bovinos, ovinos, caprinos (Capra aegagrus hircus), cerdos y aves presentes en el sistema (promedio anual) que son convertidos en unidades de ganadería, la cual está expresada en euros. El trabajo se describe en unidades de trabajo anual AWU (sigla en inglés, annual work unit). La AWU corresponde al trabajo realizado por una persona empleada por tiempo completo en una explotación ganadera [12]. En tanto, la variable alimento corresponde al alimento de cerdos y aves expresado en euros. Finalmente, la variable otros insumos comprende otros costos específicos del ganado que incluye la asesoría veterinaria, la compra ocasional de animales y otros costos de producción. Todos los valores monetarios están expresados en euros del 2015.

\section{RESULTADOS Y DISCUSIÓN}

Se estimaron diferentes tipos de funciones, siendo las tipo CobbDouglas y Translog las más utilizadas para este tipo de análisis $[6,20,27]$. En este caso, la función Translog para ambos países otorga los mejores resultados. No obstante, existen diferencias entre los países utilizando diferentes test para determinar si las ineficiencias son significativas, así como también, la inclusión o no inclusión de la variable tiempo. Los resultados de estos test se muestran en la TABLA II para Polonia y Dinamarca. Respecto a Polonia, se puede concluir que el mejor modelo es una función con la inclusión de ineficiencias, específicamente, una de tipo Translog. En el análisis, se excluye la variable tiempo al no ser significativa. En el caso de Dinamarca, la inclusión de las ineficiencias otorga mejores resultados por medio de una función Translog incluyendo la variable tiempo.

La TABLA III presenta los coeficientes estimados del modelo de frontera estocástica de producción (MFEP) con forma funcional tipo Translog para la muestra de productores porcinos de Polonia. Se observa que las estimaciones de las elasticidades de producción en las medias de los términos lineales son positivas y estadísticamente significativas al nivel del $1 \%$ o del $5 \%$.

El efecto más importante corresponde al coeficiente del alimento (Infeed), con un valor de 0,363 , lo que significa que al aumentar $1 \%$ el factor productivo alimento, manteniendo los otros factores constantes, la producción porcina aumentará en 0,363 \%. El segundo en importancia es el capital2 (Incap2) con un valor de 0,289 , es decir, si aumenta en $1 \%$ el capital 2 la producción porcina aumentará en $0,289 \%$ dejando todos los otros factores constantes (ceteris paribus). Les siguen en importancia el capital1 (Incap1), otros insumos (Inoi) y el trabajo (Inlabor) con valores de 0,167, 0,155 y 0,081 , respectivamente.

La tasa anual (constante) de progreso tecnológico es significativa al nivel del $1 \%$, indicando un crecimiento tecnológico anual de $2,1 \%$ en la muestra de productores de Polonia. La sumatoria de las elasticidades de producción entrega el valor de la elasticidad de escala. La suma es levemente superior a la unidad (1,05499), lo que indica que la tecnología presenta rendimientos ligeramente crecientes de escala. Se aplicó una prueba estadística para probar si la elasticidad de escala difiere significativamente de uno (rendimientos constantes a escala). Dado que el valor de $t(2,144)$, es mayor que el t crítico $(1,965)$, y el $P$-valor $(0,035)$, es menor al nivel de significancia del $5 \%$, se rechaza la hipótesis nula de retornos constantes de escala y se concluye que la tecnología presenta retornos crecientes de escala (TABLA IV).

El parámetro gamma $(\mathrm{Y})$ es significativo al nivel del $1 \%$ y presenta un valor de 0,715 (TABLA III), lo que indica que, tanto el término de error aleatorio como la ineficiencia son importantes para explicar desviaciones desde la función de producción. El valor de gammaVar muestra que alrededor del 47,7 \% (TABLA III) de la varianza total es debido a la ineficiencia.

Para la muestra de productores de Dinamarca, la TABLA III presenta los coeficientes estimados del MFEP con forma funcional Translog. A diferencia del caso de Polonia, en este modelo se incluye la variable cambio de eficiencia en el tiempo (time). Al aplicar un likelihood ratio test se encontró que esta forma funcional fue significativamente mejor que el modelo sin esta variable para explicar las variaciones de la producción porcina. Los signos de los coeficientes lineales estimados son como se esperaba, es decir, positivos y significativos al nivel del $1 \%$, excepto para el coeficiente del cambio tecnológico que no es significativo.

Al igual que en el modelo de Polonia, los efectos más importantes correspondieron al coeficiente del alimento (Infeed) y al del 
TABLA I

Descripción de variables utilizadas, Polonia y Dinamarca

\begin{tabular}{|c|c|c|c|c|c|c|c|c|c|c|c|}
\hline \multirow[b]{2}{*}{ Item } & \multirow[b]{2}{*}{ Año } & \multicolumn{5}{|c|}{ Polonia } & \multicolumn{5}{|c|}{ Dinamarca } \\
\hline & & Mínimo & Mediana & Media & Máximo & $\begin{array}{c}\text { Desviación } \\
\text { estándar }\end{array}$ & Mínimo & Mediana & Media & Máximo & $\begin{array}{c}\text { Desviación } \\
\text { estándar }\end{array}$ \\
\hline \multirow{6}{*}{$\begin{array}{c}\text { Producción } \\
\text { de cerdo } \\
\text { (en miles } \\
\text { de Euros) }\end{array}$} & 2010 & 26,80 & 137,84 & 186,09 & 983,75 & 165,09 & 502,89 & 1489,28 & 1726,27 & 3665,16 & 894,33 \\
\hline & 2011 & 26,54 & 128,02 & 234,42 & 2492,10 & 388,70 & 400,64 & 1234,02 & 1466,76 & 3665,88 & 762,29 \\
\hline & 2012 & 24,15 & 136,56 & 214,94 & 1727,83 & 298,51 & 408,64 & 1229,60 & 1416,84 & 3799,17 & 748,08 \\
\hline & 2013 & 21,80 & 140,01 & 226,84 & 2414,85 & 370,44 & 248,01 & 1190,48 & 1437,86 & 4009,68 & 824,67 \\
\hline & 2014 & 33,98 & 160,64 & 224,41 & 1746,12 & 279,38 & 262,27 & 1233,70 & 1473,83 & 4845,18 & 909,85 \\
\hline & 2015 & 28,06 & 159,96 & 273,57 & 2190,39 & 388,08 & 247,55 & 1148,39 & 1397,32 & 5405,99 & 940,33 \\
\hline \multirow{6}{*}{ Trabajo } & 2010 & 1,00 & 2,00 & 2,70 & 12,91 & 2,07 & 1,60 & 4,67 & 5,83 & 14,53 & 3,33 \\
\hline & 2011 & 1,00 & 2,00 & 2,75 & 13,36 & 2,28 & 1,00 & 4,09 & 4,73 & 13,00 & 2,42 \\
\hline & 2012 & 1,00 & 2,04 & 2,77 & 13,23 & 2,41 & 1,00 & 4,60 & 4,93 & 12,96 & 2,41 \\
\hline & 2013 & 1,00 & 2,00 & 2,72 & 13,32 & 2,38 & 1,00 & 4,62 & 5,30 & 13,78 & 2,79 \\
\hline & 2014 & 1,00 & 2,00 & 2,75 & 12,32 & 2,24 & 1,05 & 4,69 & 5,51 & 13,00 & 2,84 \\
\hline & 2015 & 1,00 & 2,07 & 2,84 & 12,32 & 2,36 & 1,01 & 4,30 & 5,22 & 15,00 & 2,85 \\
\hline \multirow{6}{*}{$\begin{array}{l}\text { Alimento } \\
\text { (en Euros) }\end{array}$} & 2010 & 10,23 & 75,21 & 103,03 & 350,44 & 82,68 & 263,24 & 615,33 & 796,98 & 1764,79 & 467,60 \\
\hline & 2011 & 11,99 & 72,52 & 129,25 & 1363,62 & 212,26 & 216,81 & 590,82 & 710,63 & 2075,24 & 409,60 \\
\hline & 2012 & 9,15 & 84,14 & 125,19 & 1002,08 & 165,09 & 144,94 & 615,02 & 687,37 & 2063,60 & 413,95 \\
\hline & 2013 & 11,70 & 66,90 & 124,93 & 1423,28 & 188,56 & 118,14 & 604,00 & 697,54 & 2271,89 & 459,96 \\
\hline & 2014 & 12,41 & 88,61 & 128,98 & 1455,53 & 201,44 & 111,49 & 580,78 & 726,94 & 2827,29 & 535,03 \\
\hline & 2015 & 8,35 & 84,94 & 132,79 & 1048,27 & 181,86 & 129,85 & 553,00 & 670,44 & 2476,64 & 485,99 \\
\hline \multirow{6}{*}{$\begin{array}{l}\text { Capital } 1 \\
\text { (en miles } \\
\text { de Euros) }\end{array}$} & 2010 & 47,54 & 214,75 & 279,09 & 1047,53 & 219,19 & 186,15 & 2157,14 & 2595,26 & 7616,69 & 1880,18 \\
\hline & 2011 & 24,30 & 188,00 & 274,96 & 2052,18 & 305,86 & 153,89 & 1705,85 & 2032,33 & 7052,05 & 1454,40 \\
\hline & 2012 & 20,96 & 203,45 & 267,08 & 1865,90 & 269,75 & 6,61 & 1448,25 & 1789,36 & 6545,81 & 1370,64 \\
\hline & 2013 & 18,63 & 225,07 & 262,03 & 1801,80 & 246,40 & 2,67 & 1315,18 & 1725,51 & 6164,36 & 1277,61 \\
\hline & 2014 & 17,29 & 239,58 & 255,68 & 898,12 & 185,04 & 5,88 & 1098,07 & 1569,62 & 6304,92 & 1301,84 \\
\hline & 2015 & 15,79 & 245,81 & 283,89 & 1643,79 & 250,41 & 1,33 & 902,58 & 1230,04 & 4570,83 & 1026,63 \\
\hline \multirow{6}{*}{$\begin{array}{l}\text { Capital } 2 \\
\text { (en miles } \\
\text { de Euros) }\end{array}$} & 2010 & 4,21 & 49,12 & 61,27 & 358,57 & 59,40 & 184,95 & 543,14 & 600,35 & 1234,01 & 316,50 \\
\hline & 2011 & 3,29 & 41,10 & 63,50 & 610,18 & 95,97 & 164,79 & 477,98 & 537,41 & 1376,07 & 279,23 \\
\hline & 2012 & 4,16 & 34,41 & 61,73 & 430,51 & 81,19 & 155,50 & 442,73 & 511,00 & 1388,34 & 279,33 \\
\hline & 2013 & 5,30 & 36,36 & 62,13 & 459,90 & 82,85 & 85,72 & 455,62 & 540,10 & 1390,86 & 287,88 \\
\hline & 2014 & 6,51 & 51,37 & 68,16 & 423,15 & 74,99 & 49,19 & 469,31 & 556,87 & 1412,40 & 300,18 \\
\hline & 2015 & 5,86 & 50,46 & 75,93 & 456,26 & 90,07 & 15,54 & 428,38 & 510,77 & 1570,23 & 289,23 \\
\hline \multirow{6}{*}{$\begin{array}{c}\text { Otros } \\
\text { insumos } \\
\text { (en miles } \\
\text { de Euros) }\end{array}$} & 2010 & 2,67 & 16,61 & 25,50 & 182,10 & 30,42 & 113,17 & 329,25 & 343,80 & 807,84 & 183,81 \\
\hline & 2011 & 4,31 & 16,29 & 32,36 & 338,22 & 57,38 & 96,49 & 279,36 & 299,77 & 712,54 & 137,89 \\
\hline & 2012 & 3,21 & 15,32 & 29,86 & 315,12 & 49,95 & 79,34 & 255,53 & 289,41 & 753,23 & 149,40 \\
\hline & 2013 & 2,60 & 17,71 & 30,29 & 406,28 & 52,58 & 44,19 & 278,41 & 316,40 & 899,00 & 171,65 \\
\hline & 2014 & 2,97 & 19,17 & 28,01 & 256,82 & 36,74 & 36,44 & 280,13 & 294,08 & 790,37 & 161,38 \\
\hline & 2015 & 2,48 & 19,00 & 31,63 & 292,76 & 43,98 & 39,56 & 240,17 & 262,51 & 810,11 & 149,08 \\
\hline
\end{tabular}


TABLA II

Test para Polonia y Dinamarca

\begin{tabular}{|c|c|c|c|c|c|c|c|}
\hline Objetivo & País & Modelo & DF & LogLik & Df & Chisq & $\operatorname{Pr}(>$ Chisq $)$ \\
\hline \multirow{4}{*}{$\begin{array}{l}\text { Test para determinar } \\
\text { la significancia de } \\
\text { las ineficiencias }\end{array}$} & \multirow{2}{*}{ Polonia } & OLS & 23 & 150,06 & & & \\
\hline & & Error Components Frontier & 24 & 196,58 & 1 & 93,028 & 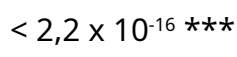 \\
\hline & \multirow{2}{*}{ Dinamarca } & OLS & 23 & 329,19 & & & \\
\hline & & Error Components Frontier & 25 & 388,69 & 2 & 119 & $<2,2 \times 10^{-16 * * *}$ \\
\hline \multirow{4}{*}{$\begin{array}{l}\text { Test de comparación entre } \\
\text { Cobb-Douglas y Translog }\end{array}$} & \multirow{2}{*}{ Polonia } & Cobb-Douglas & 10 & 165,76 & & & \\
\hline & & Translog & 24 & 196,58 & 14 & 61,63 & $6,064 \times 10^{-8 * * *}$ \\
\hline & \multirow{2}{*}{ Dinamarca } & Cobb-Douglas & 9 & 267,27 & & & \\
\hline & & Translog & 25 & 388,69 & 16 & 242,86 & $<2,2 \times 10^{-16 * \star \star}$ \\
\hline \multirow{4}{*}{$\begin{array}{l}\text { Test para medir la } \\
\text { significancia de la } \\
\text { variable tiempo }\end{array}$} & \multirow{2}{*}{ Polonia } & Translog (sin) & 24 & 196,58 & & & \\
\hline & & Translog (con) & 25 & 197,09 & 1 & 1,0125 & 0,3143 \\
\hline & \multirow{2}{*}{ Dinamarca } & Translog (sin) & 24 & 382,41 & & & \\
\hline & & Translog (con) & 25 & 388,69 & 1 & 12,565 & $0.000393 * * *$ \\
\hline
\end{tabular}

$\left({ }^{\star}\right),\left({ }^{\star *}\right),\left({ }^{(\star \star}\right)$; indican significancia de $\mathrm{P} \leq 0,10,0,05$ y 0,01 , respectivamente.

capital2 (Incap2), con valores de 0,462 y 0,264, respectivamente. Esto significa que al aumentar $1 \%$ el factor productivo alimento manteniendo los otros factores constantes, la producción porcina aumentará en $0,462 \%$, efecto superior al encontrado en el modelo de Polonia. En cambio, el efecto del capital2 (Incap2) es muy parecido, si aumenta en $1 \%$ este la producción porcina aumentará en $0,264 \%$ ceteris paribus. Les siguen en importancia el trabajo (Inlabor), otros insumos (Inoi) y el capital1 (Incap1), con elasticidades de producción en las medias de 0,145; 0,119 y 0,037 , respectivamente. Se puede observar que, en la muestra de productores de Dinamarca, el factor productivo trabajo tiene mayor efecto que en el modelo polaco.

La sumatoria de las elasticidades de producción $(1,027)$, indicó ligeros rendimientos crecientes de escala. Se aplicó una prueba estadística, cuyos resultados muestraron que el valor de $t(1,677)$, fue menor que el t crítico $(1,966)$, y el $P$-valor $(0,094)$ fue mayor que 0,05 (nivel de significancia), por lo que no se puede rechazar la hipótesis nula y se concluye que los productores de Dinamarca presentaron tecnología con rendimientos constantes de escala (TABLA IV). La tasa anual (constante) de cambio tecnológico de alrededor del $0,6 \%$ no fue significativa lo que expresa que la tecnología de producción (frontera) no cambia en el tiempo. El coeficiente estimado de la variable time fue negativo y significativo al $1 \%$, lo que significa que la eficiencia está decreciendo en el tiempo (TABLA III). En la misma Tabla, la magnitud $(0,876)$ y la significancia estadística al nivel del $1 \%$ del parámetro gamma muestraron que, tanto el término de error aleatorio como la ineficiencia, son importantes para explicar las desviaciones desde la función de producción.

Los resultados obtenidos para ambos SP son coincidentes que otros estudios desarrollados en Europa [23] y África [31]
TABLA III

Estimación frontera de posibilidades de producción Polonia y Dinamarca

\begin{tabular}{|c|c|c|}
\hline Variables & $\begin{array}{c}\text { Translog } \\
\text { (sin variable tiempo) } \\
\text { - Polonia }\end{array}$ & $\begin{array}{l}\text { Translog - } \\
\text { Dinamarca }\end{array}$ \\
\hline Intercept & $\begin{array}{l}0,074 \\
(0,022)^{\star * *}\end{array}$ & $\begin{array}{c}0,103 \\
(0,013)^{\star \star *}\end{array}$ \\
\hline Infeed & $\begin{array}{l}0,363 \\
(0,029)^{* * *}\end{array}$ & $\begin{array}{c}0,462 \\
(0,023)^{\star * *}\end{array}$ \\
\hline Inoi & $\begin{array}{l}0,155 \\
(0,029)^{\star * *}\end{array}$ & $\begin{array}{c}0,119 \\
(0,033)^{\star \star *}\end{array}$ \\
\hline Inlabor & $\begin{array}{c}0,081 \\
(0,034)^{\star \star}\end{array}$ & $\begin{array}{c}0,145 \\
(0,027)^{\star \star *}\end{array}$ \\
\hline Incap1 & $\begin{array}{l}0,167 \\
(0,029)^{\star \star \star}\end{array}$ & $\begin{array}{c}0,037 \\
(0,013)^{\star \star \star}\end{array}$ \\
\hline Incap2 & $\begin{array}{l}0,289 \\
(0,033)^{\star \star *}\end{array}$ & $\begin{array}{c}0,264 \\
(0,042)^{\star \star \star}\end{array}$ \\
\hline mYear & $\begin{array}{c}0,021 \\
(0,005)^{\star * *}\end{array}$ & $\begin{array}{c}0,006 \\
(0,005)\end{array}$ \\
\hline $0,5^{\star}(\text { Infeed })^{2}$ & $\begin{array}{l}0,436 \\
(0,081)^{\star * *}\end{array}$ & $\begin{array}{c}0,454 \\
(0,085)^{\star \star \star}\end{array}$ \\
\hline $0,5 *(\text { Inoi })^{2}$ & $\begin{array}{c}0,070 \\
(0,067)\end{array}$ & $\begin{array}{l}-0,152 \\
(0,18)\end{array}$ \\
\hline $0,5 *(\text { Inlabor })^{2}$ & $\begin{array}{c}0,061 \\
(0,116)\end{array}$ & $\begin{array}{c}0,044 \\
(0,104)\end{array}$ \\
\hline $0,5^{*}(\operatorname{Incap} 1)^{2}$ & $\begin{array}{c}0,085 \\
(0,067)\end{array}$ & $\begin{array}{c}0,014 \\
(0,006)^{\star \star}\end{array}$ \\
\hline
\end{tabular}


TABLA III (cont...)

Estimación frontera de posibilidades de producción Polonia y Dinamarca

\begin{tabular}{|c|c|c|}
\hline $0,5 *(\operatorname{Incap} 2)^{2}$ & $\begin{array}{l}0,236 \\
(0,055)^{* * *}\end{array}$ & $\begin{array}{c}0,062 \\
(0,106)\end{array}$ \\
\hline Infeed * Inoi & $\begin{array}{c}-0,072 \\
(0,054)\end{array}$ & $\begin{array}{l}-0,151 \\
(0,083)^{*}\end{array}$ \\
\hline Inoi * Inlabor & $\begin{array}{l}-0,021 \\
(0,059)\end{array}$ & $\begin{array}{l}-0,011 \\
(0,096)\end{array}$ \\
\hline Infeed * Inlabor & $\begin{array}{l}-0,114 \\
(0,065)^{\star}\end{array}$ & $\begin{array}{l}-0,09 \\
(0,068)\end{array}$ \\
\hline Incap2 * Infeed & $\begin{array}{l}-0,296 \\
(0,058)^{\star * *}\end{array}$ & $\begin{array}{l}-0,338 \\
(0,083)^{\star * *}\end{array}$ \\
\hline Incap2 * Inlabor & $\begin{array}{c}0,093 \\
(0,065)\end{array}$ & $\begin{array}{l}0,109 \\
(0,11)\end{array}$ \\
\hline Incap2 * Inoi & $\begin{array}{c}0,037 \\
(0,049)\end{array}$ & $\begin{array}{c}0,299 \\
(0,16)^{*}\end{array}$ \\
\hline Incap1 * Incap2 & $\begin{array}{c}0,068 \\
(0,049)\end{array}$ & $\begin{array}{l}-0,054 \\
(0,043)\end{array}$ \\
\hline Incap1 * Infeed & $\begin{array}{c}-0,013 \\
(0,051)\end{array}$ & $\begin{array}{l}-0,003 \\
(0,026)\end{array}$ \\
\hline Incap1 * Inoi & $\begin{array}{l}-0,078 \\
(0,057)\end{array}$ & $\begin{array}{c}0,02 \\
(0,02)\end{array}$ \\
\hline Incap1 * Inlabor & $\begin{array}{c}0,003 \\
(0,059)\end{array}$ & $\begin{array}{l}-0,006 \\
(0,03)\end{array}$ \\
\hline sigmaSq & $\begin{array}{l}0,054 \\
(0,008)^{\star \star *}\end{array}$ & $\begin{array}{c}0,034 \\
(0,007)^{\star * *}\end{array}$ \\
\hline Gamma & $\begin{array}{l}0,715 \\
(0,052)^{\star \star *}\end{array}$ & $\begin{array}{l}0,876 \\
(0,029)^{\star * *}\end{array}$ \\
\hline Time & - & $\begin{array}{l}-0,13 \\
(0,037)^{\star \star *}\end{array}$ \\
\hline Lambda & $\begin{array}{c}1,585 \\
(0,200)^{\star \star *}\end{array}$ & $\begin{array}{l}2,662 \\
(0,355)^{\star \star *}\end{array}$ \\
\hline GammaVar & 0,477 & - \\
\hline
\end{tabular}

Los errores estándar (EE) de las estimaciones se muestran entre paréntesis. $\left({ }^{*}\right),(* \star),(* \star \star)$; indican significancia de $P \leq 0,10,0,05 y$ 0,01 , respectivamente.

TABLA IV

Test retorno a escala constante Polonia y Dinamarca

\begin{tabular}{|c|c|c|c|c|c|c|}
\hline \multirow{2}{*}{ País } & \multirow{2}{*}{$\begin{array}{l}\text { Sumatoria } \\
\text { coeficientes }\end{array}$} & \multirow{2}{*}{$\begin{array}{c}\text { Valor } \\
\mathbf{t}\end{array}$} & \multirow{2}{*}{$\begin{array}{l}\text { Valor } \\
\text { crítico }\end{array}$} & \multirow{2}{*}{$\begin{array}{c}\text { Valor } \\
\mathbf{p}\end{array}$} & \multicolumn{2}{|c|}{$\begin{array}{c}\text { Intervalo de } \\
\text { confianza }(95 \%)\end{array}$} \\
\hline & & & & & $\begin{array}{c}\text { Límite } \\
\text { inferior }\end{array}$ & $\begin{array}{c}\text { Límite } \\
\text { superior }\end{array}$ \\
\hline Polonia & 1,055 & 2,145 & 1,966 & 0,033 & 1,005 & 1,105 \\
\hline Dinamarca & 1,027 & 1,677 & 1,966 & 0,094 & 0,995 & 1,059 \\
\hline
\end{tabular}

que analizaron la contribución de las variables mano de obra y alimentación en la eficiencia de un SP porcino.

La eficiencia técnica promedio para los productores de Polonia (TABLA V) de esta muestra llegó a 0,859 con una mediana de 0,889. La FIG. 1 muestra la distribución de la eficiencia técnica de los productores, donde se observa una distribución asimétrica de la eficiencia con una cola hacia la izquierda, con mayor cantidad de productores en el rango de eficiencias superiores a 0,85.

TABLA V

Resumen eficiencias Polonia

\begin{tabular}{cccc}
\hline Mínimo & Mediana & Media & Máximo \\
\hline 0,644 & 0,889 & 0,859 & 0,971 \\
\hline
\end{tabular}

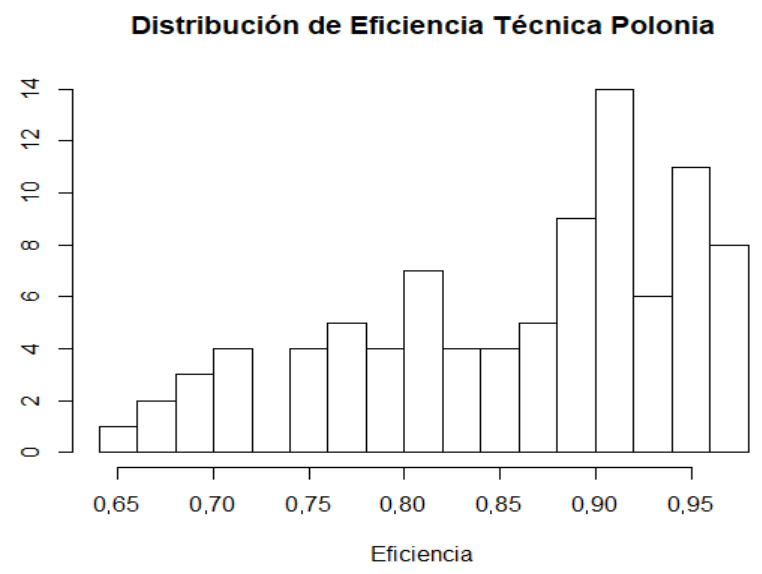

Distribución de Eficiencia Técnica Dinamarca

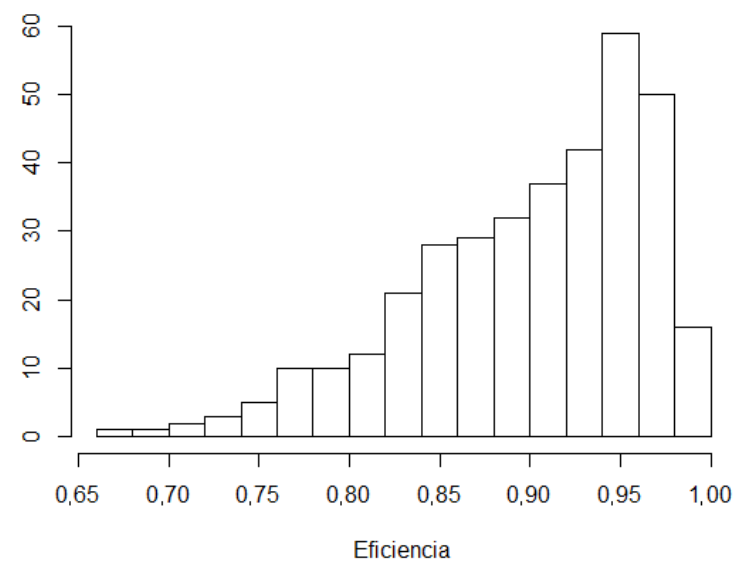

FIGURA 1. Distribución de eficiencia técnica en Polonia y Dinamarca 
La eficiencia técnica promedio de esta muestra de productores porcinos de Dinamarca llegó a 0,899 , lo que es un valor alto y superior al encontrado para los productores de Polonia. Este hecho es coincidente con lo establecido por algunos autores en su análisis sobre el sistema productivo porcino europeo [12, 29]. La distribución de las eficiencias presenta una gran concentración de los productores con eficiencias en los rangos superiores y pocos en la porción inferior del rango, esto genera una distribución con una cola hacia la izquierda, lo cual se puede observar en la FIG. 1.

Además, la TABLA VI muestra un resumen estadístico de las eficiencias por año para la muestra de productores de Dinamarca. Es clara la disminución de la eficiencia media en el periodo de estudio, desde 0,937 el año 2010 hasta 0,899 el año 2015. Esta situación puede ser explicada por la ampliación del rango de valores de la eficiencia, especialmente, debido a la disminución del valor mínimo desde 0,863 hasta 0,667 . Además, lo observado es consistente con el signo y la significancia del coeficiente de la variación de la eficiencia en el tiempo.

TABLA VI

Resumen eficiencias productores Dinamarca por año

\begin{tabular}{ccccc}
\hline Año & Mínimo & Mediana & Media & Máximo \\
\hline 2010 & 0,863 & 0,937 & 0,932 & 0,990 \\
2011 & 0,771 & 0,928 & 0,918 & 0,993 \\
2012 & 0,744 & 0,920 & 0,910 & 0,993 \\
2013 & 0,714 & 0,904 & 0,896 & 0,985 \\
2014 & 0,681 & 0,896 & 0,884 & 0,982 \\
2015 & 0,668 & 0,899 & 0,876 & 0,989 \\
\hline
\end{tabular}

\section{CONCLUSIONES}

El mercado de la $\mathrm{CdC}$ en las últimas décadas está creciendo hasta convertirse en el segundo tipo de carne producido en el mundo. Dentro de este competitivo mercado, Europa es la segunda región en importancia productiva después de Asia. Dos actores relevantes en el mercado europeo son Dinamarca y Polonia. A pesar de que ambos países están realizando cambios de tal forma de adaptarse de manera adecuada a los cambios en el mercado porcino, éstos presentan dispares estados de desarrollo con algunas diferencias estructurales en sus SP de CdC. Dinamarca sobresale como un país líder en tecnología y en sanidad, mientras que Polonia está en procesos de cambios que le permitan mejorar sobre todo en la integración vertical y horizontal de su cadena productiva.

Los resultados de este estudio muestran que, a pesar de las diferencias estructurales que presentan ambos países, la eficiencia técnica relativa media de los productores porcinos en Polonia y Dinamarca es media alta, concretamente de 0,859 y 0,899 , respectivamente, siendo la distribución de eficiencias asimétrica con una cola hacia la izquierda. A pesar de esto, los niveles de eficiencia podrían mejorarse si los productores realizan combinaciones óptimas de los factores productivos que beneficien la producción porcina en el territorio.

El factor productivo que presenta mayor impacto en la producción porcina en ambos países corresponde al alimento, con elasticidades de $0,363 \%$ en Polonia y $0,462 \%$ en Dinamarca. A su vez, el factor trabajo cobra mayor relevancia en Dinamarca que en Polonia, con elasticidades de 0,145 y 0,081, respectivamente, lo que transforma al trabajo en el tercer factor productivo en importancia en Dinamarca. Los factores otros insumos y capital (equipos y edificaciones) son significativos, pero con menores elasticidades de producción para ambos países.

Las muestras de productores de Polonia presentan tecnologías con rendimientos de escala levemente superior a la unidad, lo que indica rendimientos crecientes de escala. Es interesante el hecho que, los productores de Polonia presentan un progreso tecnológico anual constante de 2,1\%, sin cambio de eficiencia en el periodo. Esto puede ser el efecto de cambios estructurales en el SP, que aumentaron la productividad pero no tuvieron efectos significativos en la eficiencia. Mientras tanto, la muestra de productores de Dinamarca no mostró cambios significativos en el progreso tecnológico en el tiempo, pero si una disminución significativa de la eficiencia. Esta situación, junto con factores relacionados al mercado, podría explicar la baja en la producción de $\mathrm{CdC}$ en el periodo de estudio.

Finalmente, este estudio es importante para expandir la literatura en la eficiencia técnica de la producción porcina, sobre todo porque es la primera vez que se mide la eficiencia técnica en dos países relevantes en el mercado europeo. Sin embargo, quedan para posteriores investigaciones determinar los factores que afectan o explican el cambio en la media de la eficiencia técnica, por ejemplo, las causas que subyacen a la caída en la eficiencia en la producción porcina en Dinamarca, la que pasó de 0,937 en 2010 a 0,899 en 2015, y la incorporación del riesgo de producción en el análisis. Esto permitiría enfocar los aspectos de perfeccionamiento en la labor de los productores porcinos, y a su vez, generar y aplicar políticas públicas de apoyo a los productores que contribuyan a mejorar su competitividad y recuperar los niveles de producción y eficiencia de inicio de la década.

\section{AGRADECIMIENTOS}

Los autores agradecen a la European Commission, DirectorateGeneral Agriculture, Unit AGRI.G.3 por proporcionar los datos FADN (Farm Accountancy Data Network) para este análisis.

\section{REFERENCIAS BIBLIOGRAFICAS}

[1] AIGNER, D. C.; LOVELL, A. K.; SCHMIDT, P. Formulation and estimation of stochastic frontier production function models. J. Econometr. 6(1): 21-37. 1977.

[2] ASMILD, M.; HOUGAARD, J. L.; KRONBORG, D.; KVIST, H. K. Measuring inefficiency via potential improvements. J. Product. Analy.19(1): 59-76. 2003.

[3] BATTES.E, G.E.; COELLI, T.J. Frontier production functions, technical efficiency and panel data: With application to paddy farmers in India. J. Product. Analy. 3: 153-169. 1992. 
[4] BATtese, G. E.; COELLI, T. J. A model for technical inefficiency effects in a stochastic frontier production function for panel data. Empiric. Economics. 20: 325-32. 1995.

[5] BOGETOFT, P.; OTTO, L. Stochastic Frontier Analysis SFA. Benchmarking with Dea, Sfa, and R. Springer Science \& Business Media. Pp. 197-232. 2010.

[6] BRAVO-URETA, B.; SOLIS, D.; MOREIRA, V.; MARIPANI, J.; ABDOURAHMANE, T.; RIVAS, T. Technical efficiency in farming: a meta-regression analysis. J. Product. Analy. 27: 57-72. 2007.

[7] CALAFAT, C.; MARTÍ, M.; PUERTAS, R. Eficiencia del sector ganadero valenciano: Aproximación no paramétrica. ITEA Inform. Tecn. Econom. Agra.114(1): 61-77. 2018.

[8] CHAITIP, P.; CHAIBOONSRI, C.; INLUANG, F. The Production of Thailand's Sugarcane: Using Panel Data Envelopment Analysis (Panel DEA) Based Decision on Bootstrapping Method. Procedia Econom. and Finance. 14: 120-127. 2014.

[9] COELLI, T.; HENNINGSEN, A. Frontier: Stochastic Frontier Analysis. R package version 1.1-8. On line: https://bit.ly/3cnP3EC. 2020.

[10] COELLI, T. J.; RAO, D. S. P.; O'DONNELL, C. J.; BATTESE, G. E. Stochastic Frontier Analysis. An introduction to efficiency and productivity analysis. Springer Science \& Business Media Inc. Pp. 241-261. 2005.

[11] DANISH AGRICULTURE AND FOOD COUNCIL (DAFC). Fakta Om Erhvervet 2017: Fødevareklyngen Har Kurs Mod Fremtiden (Facts and figures 2017, the food cluster is heading for the future). Copenhague. 40 pp. 2018.

[12] OFICINA ESTADÍSTICA DE LA UNIÓN EUROPEA (EUROSTAT). Agricultural production - livestock and meat - Statistics Explained. 2020. On-line: https://bit.ly/3yYtQuq. 10.11.2020.

[13] FARRELL, MJ. The measurement of productive efficiency. J. Royal Statist. Soc. 120(A): 253-281. 1957.

[14] ORGANIZACIÓN DE LAS NACIONES UNIDAS PARA LA ALIMENTACIÓN Y LAAGRICULTURA (FAO) Y MINISTERIO DE MEDIO AMBIENTE Y ALIMENTACIÓN DE DINAMARCA - ADMINISTRACIÓN DANESA DE VETERINARIA Y ALIMENTACIÓN. Hacer frente al uso de antimicrobianos y la resistencia a los antimicrobianos en la producción porcina: lecciones aprendidas de Dinamarca. Roma. 52 pp. 2019.

[15] FOOD AND AGRICULTURE ORGANIZATION OF THE UNITED NATION (FAO). Food Outlook - Biannual Report on Global Food Markets: June 2020. Food Outlook. 1. 160 pp. 2020.

[16] GARCÍA, E.; SERRANO, C. Competitividad y eficiencia. Estud. Econom. Aplicad. 21(3): 423-450. 2003.

[17] HENNINGSEN, A. Introduction to Econometric Production Analysis with R. Collection of Lecture Notes. 2nd Draft Version. Department of Food and Resource Economics, University of Copenhagen. 370 pp. 2018.

[18] HENNINGSEN, A.; CZEKAJ, T. G.; FORKMAN, B.; LUND, M; NIELSEN, A. S. The relationship between animal welfare and economic performance at farm level: A quantitative study of Danish pig producers. J. Agric. Econom. 69(1): 142-162. 2018.
[19] HOMAN, M. Structural changes in the Polish pig sector. 2018. Embassy of the Kingdom of the Netherlands. On-line: https:// www.agroberichtenbuitenland.nl. 10.01.2021.

[20] JAIME, M.; SALAZAR, C. Participation in Organizations, Technical Efficiency and Territorial Differences: A Study of Small Wheat Farmers in Chile. Chilean J. Agric. Res. 71(1): 104-113. 2011.

[21] KUMBHAKAR, S.; LOVELL, C. The Estimation of Technical Efficiency. Stochastic Frontier Analysis. Cambridge: Cambridge University Press. Pp. 63-130. 2000.

[22] KUMBHAKAR, S.; WANG, H.; HORNCASTLE, A. Estimation of Technical Efficiency in Single Equation Panel Models. A Practitioner's Guide to Stochastic Frontier Analysis Using Stata. Cambridge: Cambridge University Press. Pp.241-278. 2015.

[23] LABAJOVA, K.; HANSSON, H.; ASMILD, M.; GÖRANSSON, L.; LAGERKVIST, C. J.; NEIL, M. Multidirectional analysis of technical efficiency for pig production systems: The case of Sweden. Livest. Sci. 187: 168-180. 2016.

[24] MEEUSEN, W.; VAN DEN BROECK, J. Efficiency Estimation from Cobb-Douglas Production Functions with Composed Error. Intern. Econom. Rev. 18(2): 435-444. 1977.

[25] MINISTERIO DE AGRICULTURA, PESCA Y ALIMENTACIÓN, GOBIERNO DE ESPAÑA. El sector de la Carne de Cerdo en cifras: Principales Indicadores Económicos. Subdirección General de Producciones Ganaderas y Cinegéticas, Dirección General de Producciones y Mercados Agrarios. 63 pp. 2020.

[26] MROCZEK, R. Red meat sector after accession of Poland to the EU. Econom. Sociol. 2(2): 69-76. 2009.

[27] ORTEGA, L.; WARD, R.; ANDREW, C. Technical Efficiency of the Dual-Purpose Cattle System in Venezuela. J. Agric. Appl. Econom. 39(3):719-733. 2007.

[28] R CORE TEAM. R: A language and environment for statistical computing. R Foundation for Statistical Computing, Vienna, Austria. On Line: https://www.R-project.org/. 2020.

[29] ROGUET, C.; GAIGNÉ, C.; CARIOU, S.; CARLIER, M.; CHATELLIER, V.; CHENUT, R.; DANIEL, K.; PERROT, C. Spécialisation territoriale et concentration des productions animales européennes: état des lieux et facteurs explicatifs. INRA Prod. Anim. 28(1): 5-22. 2015.

[30] TIAN, X.; SUN, F.; ZHOU, Y. Technical efficiency and its determinants in China's hog production. J. Integrat. Agric. 14(6): 1057-1068. 2015.

[31] UMEH, J. C.; OGBANJE, C.; ADEJO, M. A. Technical efficiency analysis of pig production: A sustainable animal protein augmentation for Nigerians. J. Advanc. Agric. Technol. 2(1): 19-24. 2015

[32] WAGAN, S.A.; MEMON, Q.U.A.; QIAN, L.; JINGDONG, $\mathrm{L}$. Measuring the efficiency of Pakistani rice production via stochastic frontier and data envelopment analyses. Custos e @gronegócio on line. 15(2): 63-86. 2019. 